\section{Xpress sequencing for express screening}

\author{
By Kai-Jye Lou, Staff Writer
}

Researchers at the University of California, San Diego and the Gladstone Institute of Cardiovascular Disease have developed a system for genomic-based drug screening that combines mRNA profiling and an automated, high throughput sequencing platform. ${ }^{1}$ Startup 255Xpress Inc. has licensed the technology and is adapting it for a variety of drug screening assays.

A team led by Xiang-Dong Fu, professor of cellular and molecular medicine at UCSD and a cofounder of 255Xpress, described an mRNA profiling technique in 2002 that allowed for parallel analysis of mRNA transcripts on microarrays without the need for RNA purification or cDNA synthesis. ${ }^{2}$ The approach, which involved RNA annealing, selection and ligation, was useful for quantifying gene expression and mRNA isoforms. It also led to the development of genotyping assays marketed by Illumina Inc.
According to $\mathrm{Fu}$, although at the time it was clear that mRNA profiling strategies also could be applied in principle to drug screening, his group's microarray-based system would have had limited throughput and potentially caused artifacts.

Now, Fu and collaborators at Gladstone have addressed these issues by scrapping the microarray component altogether and have instead combined a platform that fully automates mRNA profiling with a high throughput sequencing platform that uses bar-coded primers and deep sequencing to quantify gene expression.

The resulting system, which was implemented by combining Beckman Coulter Inc.'s Biomek FX laboratory automation workstation with Illumina's HiSeq2000 molecular sequencing system, can help screen entire compound libraries for molecules that modulate the expression of diseaseassociated genes and can simultaneously probe those molecules for potential toxicity and selectivity issues (see Figure 1, "High throughput sequencing for drug discovery").

As proof of concept, the group screened a library of about 4,000 compounds for molecules that had activity against a panel of 70 androgen-associated genes but lacked activity against 30 housekeepingand cytotoxicity-related genes.

Follow-up in vitro validation studies showed the top three hits from the screen - the cardiac glycosides digoxin, peruvoside and strophanthidin-inhibited the growth of both castration-sensitive and castration-resistant prostate cancer cell lines at nanomolar concentrations. Additional cellular assays on the most potent compound, peruvoside, showed that it induced androgen receptor degradation and inhibited the growth of castration-resistant prostate cancer cells without triggering a general cytotoxic response.
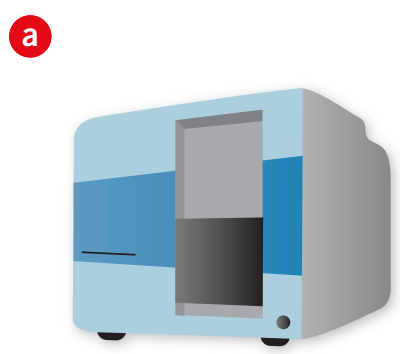

Use automated system to add cells to wells within plates, add compound to the wells and generate cell lysates

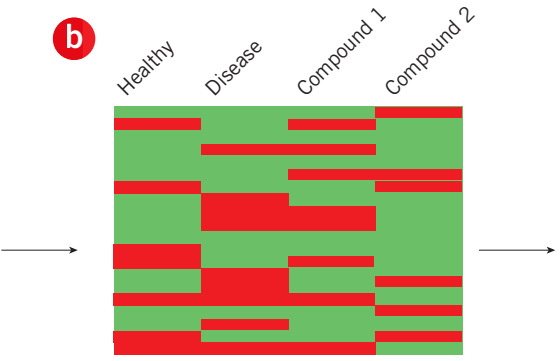

Use molecular sequencer to quantify gene expression changes in response to library compounds

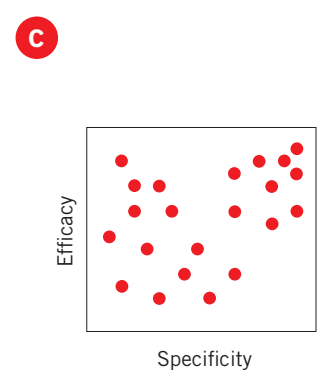

Rate compounds based on their ability to modulate gene expression in desired manner

Figure 1. High throughput sequencing for drug discovery. As reported in Li et al., a high throughput sequencing platform could aid the discovery of compounds that reverse gene expression changes associated with disease.

In the method, a profile is generated of gene expression changes associated with a disease state. A range of housekeeping- and cytotoxicity-related genes are incorporated into the panel to control for a compound's specificity.

Target cells are loaded into the wells of a microtiter plate, and a different library compound is added to each well [a]. Cells are then lysed, and the resulting lysate is profiled and transferred to a next-generation sequencing system for sequencing and quantification [b]. Compounds are evaluated based on their ability to modulate the expression of disease-associated genes without significantly affecting the expression of housekeeping- and cytotoxicity-related genes [c].

The high throughput nature of the approach allows researchers to screen libraries for compounds that correct disease-linked genetic signatures. 
Results were reported in the Proceedings of the National Academy of Sciences.

"Instead of microarrays, we were now able to use our strategy with an automated molecular sequencer to quantify gene expression," said co-corresponding author Fu. "This was the key transition to achieve the high throughput needed for screening applications."

"The ability to use bar-coded primers enables many samples to be pooled prior to sequencing, which dramatically increases the throughput," added Ultan McDermott, a career development fellow group leader in the Cancer Genome Project at the Wellcome Trust Sanger Institute and a practicing medical oncologist at Addenbrooke's Hospital.

The other co-corresponding authors were Michael Rosenfeld, a professor of medicine at UCSD, and Sheng Ding, a senior investigator at Gladstone and a professor of pharmaceutical chemistry at the University of California, San Francisco.

\section{Getting a full picture}

Fu said his group's technology could address some of the shortcomings of the two main classes of screening platforms used by pharmas: phenotypic and target based.

"With phenotypic screens, one won't know what the molecular target is, and many approaches based on phenotypic screening techniques

"The ability to use barcoded primers enables many samples to be pooled prior to sequencing, which dramatically increases the throughput."

- Ultan McDermott,

Wellcome Trust Sanger Institute such as behavior-based assays are difficult to carry out in high throughput fashion," he told SciBX. "In target-based screening, one may know the biology of the target on target cells but not necessarily in other cells, so one will want to carry out many counter screens that will consume a lot of resources and time."

Fu said his group's approach can simultaneously monitor multiple groups of responses to a particular compound, such as those related to efficacy and toxicity. He added that the approach also can establish links between a disease phenotype and genotype, which could aid the discovery of new therapeutic targets and disease mechanisms.

"Even in the case of complex diseases, where you may not know what you want to target, you could look at the overall gene expression profile and use it to screen for molecules that modulate it in a manner that could be associated with improvement of the disease phenotype," Fu said.

Finally, as commercial sequencing systems get cheaper and faster, Fu expects the number of genes that could be probed at the same time will increase and the costs associated with using the screening platform will fall.

His group is seeking to expand the use of the screening approach for both basic and translational science research, for example, by using it to identify chemical probes to dissect disease-related pathways and gene networks.

The University of California and Illumina jointly patented the profiling technology used in the screening platform, but the company returned its exclusive rights to the University of California system after transitioning to a different platform for genotyping. The University of California has licensed the technology to 255Xpress. Fu said several undisclosed pharmas have expressed interest in the technology and that some already are working with 255Xpress to test the platform.

The biotech is using the technology to develop gene expression analysis assays for drug screening applications.

Lou, K.-J. SciBX 5(14); doi:10.1038/scibx.2012.354

Published online April 5, 2012

\section{REFERENCES}

1. Li, H. et al. Proc. Natl. Acad. Sci. USA; published online March 5, 2012; doi:10.1073/pnas.1200305109

Contact: Xiang-Dong Fu, University of California, San Diego, La Jolla, Calif.

e-mail: xdfu@ucsd.edu

Contact: Sheng Ding, Gladstone Institute of Cardiovascular Disease, San Francisco, Calif.

e-mail: sheng.ding@gladstone.ucsf.edu

Contact: Michael G. Rosenfeld, University of California, San Diego, La Jolla, Calif.

e-mail: mrosenfeld@ucsd.edu

2. Yeakley, J.M. et al. Nat. Biotechnol. 20, 353-358 (2002)

\section{COMPANIES AND INSTITUTIONS MENTIONED}

255Xpress Inc., San Diego, Calif.

Addenbrooke's Hospital, Cambridge, U.K.

Beckman Coulter Inc. (NYSE:BEC), Brea, Calif.

Gladstone Institute of Cardiovascular Disease,

San Francisco, Calif.

Illumina Inc. (NASDAQ:ILMN), San Diego, Calif.

University of California, Oakland, Calif.

University of California, San Diego, La Jolla, Calif.

University of California, San Francisco, Calif.

Wellcome Trust Sanger Institute, London, U.K. 